\title{
Study on Growth Monitoring and Yield Prediction of Winter Wheat in the South of Shanxi Province Based on MERSI Data and ALMANAC Crop Model
}

\author{
Dong Xiang1,2, Shuying Bai', Xiaonan $\mathrm{Mi}^{3}$, Yongqiang Zhao3, Mengwei $\mathrm{Li}^{3}$ \\ ${ }^{1}$ Nanjing University of Information Science \& Technology, Nanjing, China \\ ${ }^{2}$ The Meteorological Bureau of Shanxi Province, Taiyuan, China \\ ${ }^{3}$ Shanxi Climate Center, Taiyuan, China \\ Email: 420285541@qq.com
}

How to cite this paper: Xiang, D., Bai, S. Y., Mi, X. N., Zhao, Y. Q., \& Li, M. W. (2019). Study on Growth Monitoring and Yield Prediction of Winter Wheat in the South of Shanxi Province Based on MERSI Data and ALMANAC Crop Model. Journal of Geoscience and Environment Protection, 7, 1-10.

https://doi.org/10.4236/gep.2019.79001

Received: May 24, 2019

Accepted: September 7, 2019

Published: September 10, 2019

Copyright $\odot 2019$ by author(s) and Scientific Research Publishing Inc. This work is licensed under the Creative Commons Attribution International License (CC BY 4.0).

http://creativecommons.org/licenses/by/4.0/

Open Access

\begin{abstract}
Accurate crop growth monitoring and yield forecasting have important implications for food security and agricultural macro-control. Crop simulation and satellite remote sensing have their own advantages, combining the two can improve the real-time mechanism and accuracy of agricultural monitoring and evaluation. The research is based on the MERSI data carried by China's new generation Fengyun-3 meteorological satellite, combined with the US ALMANAC crop model, established the NDVI-LAI model and realized the acquisition of LAI data from point to surface. Because of the principle of the relationship between the morphological changes of LAI curve and the growth of crops, an index that can be used to determine the growth of crops is established to realize real-time, dynamic and wide-scale monitoring of winter wheat growth. At the same time, the index was used to select the different key growth stages of winter wheat for yield estimation. The results showed that the relative error of total yield during the filling period was low, nearly $5 \%$. The research results show that the combination of domestic meteorological satellite Fengyun-3 and ALMANAC crop model for crop growth monitoring and yield estimation is feasible, and further expands the application range of domestic satellites.
\end{abstract}

\section{Keywords}

FY-3 Satellite, ALMANAC Crop Model, Winter Wheat, Forecast Yield

\section{Introduction}

As one of the main food crops in Shanxi Province of China, winter wheat plays 
an important role in agricultural production. Therefore, the realization of the province's crop planting area extraction, growth monitoring and production forecast is of great significance for guiding agricultural production. Satellite remote sensing technology provides an effective means for large-scale, real-time and dynamic crop growth monitoring and yield estimation (Zhao et al., 2004), and the crop growth model can simulate the growth mechanism of crops based on the spatial time scale of a single test site and its relationship with the outside world. The relationship between environmental factors (Wang et al., 2018), combining the advantages of both will provide strong support for the monitoring and evaluation of regional crop productivity. At present, there are four main ways to combine crop growth models with remote sensing information, namely verification method (Jiang, 2012), driving method (Moulin et al., 1995), initialization/parameterization method (regulation method) (Clevers et al., 1994) and assimilation method (Guerif \& Duke, 1998). Most of the remote sensing data used in various methods are NOAA, MODIS, TM, etc. The use of domestic satellite Fengyun No. 3 MERSI data combined with crop model to study winter wheat growth monitoring and yield forecast is still rare in China. Based on the research of MERSI data to extract winter wheat planting area and the localization of American ALMANAC crop model to Shanxi localization, this paper attempts to combine the two to realize the monitoring and yield estimation of winter small growth in Shanxi Province, and then expand the domestic wind and cloudy weather. The application of satellites in agriculture is based on the previous winter wheat area extraction and ALMANAC crop model localization research, a remote sensing-crop model combined with the winter small-scale monitoring and yield prediction model was established to realize real-time and dynamic growth monitoring and yield prediction of winter wheat during the whole development period.

\section{Data and Method}

\subsection{Data}

As the main food crop in Shanxi Province, winter wheat plays an important role in agricultural production. The study area selects the main planting area of winter wheat in Shanxi Province-the southern part of Shanxi Province. The geographical position is between $110^{\circ} 15^{\prime}-113^{\circ} 37^{\prime}$ east longitude and $34^{\circ} 35^{\prime}-36^{\circ} 57^{\prime}$ north latitude. This area is a temperate continental climate with an annual average. The temperature ranges from $10.4^{\circ} \mathrm{C}$ to $13.0^{\circ} \mathrm{C}$, the frost-free period is between 160 and 210 days, and the annual average precipitation is around 600 $750 \mathrm{~mm}$. The agricultural production conditions are unique, the terrain is flat, the climate is mild, the soil is fertile, and the light is sufficient (Wu et al., 2009).

The medium resolution imaging spectrometer (MERSI) is the payload of the new generation of polar-orbiting satellite Fengyun No.3 launched in China. The channel spectrum range setting is similar to that of the MOIS mounted on the 
US EOS, but the channel with a resolution of $250 \mathrm{~m}$ below the star point is increased to 5 The MODIS $250 \mathrm{~m}$ has only two channels of data. Therefore, MERSI's classification and accuracy of features are better than MODIS numbers (Zhang, 2012; Kiniry et al., 1996).

The $250 \mathrm{~m}$ MERSI data for the winter wheat growth period of 2015 and 2016 were selected. Firstly, the data were subjected to geometric precision correction, radiation correction, projection conversion, area cropping, solar elevation correction, atmospheric correction and other pretreatment (Wu et al., 2011). Image data with a solar elevation angle greater than $60^{\circ}$ is selected as the main data source, and the normalized vegetation index NDVI (Wang et al., 2017) is calculated for the red and near infrared channels to form a vegetation index data set.

In order to accurately reflect the growth stage of winter wheat in Shanxi Province, the growth phenology data of winter wheat is needed. The main phenological period is regreening, heading, and milk ripening. Collect statistics on the area and yield of winter wheat in the various counties in the study area for the correction and assessment of monitoring results. Other data include regional land use status maps and administrative boundary vector data for each county.

\subsection{Method}

Wu et al. (2018) found that the linear mixed pixel decomposition method can obtain the percentage of winter wheat in a single pixel. This method is used to extract the winter wheat planting area of Yuncheng, the main winter wheat producing area in Shanxi. Compared with the actual winter wheat planting area, the relative error is about $3 \%$. It is significantly better than the accuracy of traditional remote sensing classification methods. Based on the research results, combined with the growth characteristics of winter wheat and other surface vegetation in the study area, the MERSI clear sky data with good quality can be selected in the regreening stage where winter wheat information can be highlighted, and the winter wheat planting area in the study area is extracted by linear mixed pixel decomposition method. In 2015, the 62 nd and 71 st synthetic data sets were selected; in 2016, the 62nd, 68th, and 86th synthetic data sets were selected.

ALMANAC (Agricultural Land Management and Numerical Assessment Criteria) model operates on a daily time step and has components to simulate crop growth and competition of plant communities, hydrology, erosion, soil carbon, nutrient cycling, and pesticide fate. The model has been extensively used to analyze plant community dynamics, phenology, water use efficiency, radiation use efficiency, crop grain and bioenergy feedstock yields, and the associated impacts on ecosystem services. It can also be adapted to evaluate impacts of climate change and global warming (Kiniry et al., 1996). The model uses leaf area index, canopy interception of solar radiation, simulates crop respiration and photosynthesis, and can simulate crop growth under nutrient stress, water stress, and temperature stress. It is developed on the basis of American crop varieties and 
growing environment. When introducing the crop growth model in other places, it is first necessary to use the local crops, meteorology, soil and other data to adapt the model parameters, correction and simulation results (Kiniry et al., 1992). Wu Yongli et al. used the ALMANAC crop growth model to simulate the winter wheat yield in high, middle and low yield fields in Hongdong County, Shanxi Province. Numerous parameters such as crop attributes, soil, meteorological and field management measures required by the model were collected, and the parameters were adjusted according to actual conditions to realize localization of crop models. The results showed that the relative error of simulated yield of winter wheat was $-7.8 \%-5.7 \%$, and the relative error of leaf area index was $-12.5 \%-13.6 \%$. The dynamic change of winter wheat yield and leaf area index could be simulated by ALMANAC model (Wu et al., 2018) fault parameter values of ALMANAC model, and are applied to the simulation of crop leaf area index and winter wheat yield in meteorological sites in the study area.

During the acquisition process of satellite remote sensing time series data, due to the discontinuity of data acquisition time and various factors, such as solar elevation angle, observation angle, and water vapor, aerosol and cloud, various land types are made. Time series data changes are irregular, and trend analysis and information extraction cannot be performed on the time dimension. In order to reduce the impact of these factors, it is necessary to reconstruct time series data (Gao \& Liu, 2000; Mohamed et al., 2004). In this paper, the time series harmonic analysis method (HANTS) is used to smooth the vegetation index time series data (Zhou et al., 2016). This method can quantitatively express the dynamic change information of vegetation by revealing the periodic law of the curve, which is an effective method for analyzing and extracting vegetation information. The core of the algorithm is to use the two-dimensional Fourier transform method to fit the variation of the NDVI curve over the entire period.

Leaf area index LAI refers to the ratio of leaf area per unit area of surface area, which can quantitatively indicate vegetation vigor, dynamically monitor crop growth, and is closely related to respiration and photosynthesis product accumulation, and is an important parameter for yield estimation (Li, 1997). In this paper, the correlation between LAI and NDVI is analyzed by the commonly used vegetation index method, and the LAI calculation model is established.

According to the principle of the relationship between leaf shape index LAI curve morphological change and crop growth (Wu et al., 2008), the real-time LAI and crop model can be compared and analyzed based on the climatic LAI simulated by historical average climate data. If the growth of a certain time period $(\mathrm{T})$ is monitored, the actual LAI value in the time period can be integrated to calculate the proportion R of the LAI integral value of the corresponding time period $(\mathrm{Tb})$ in the climatic state. Since the leaf area index of the key growth period of crops has a good correlation with crop yields (Ren et al., 2010), R is applied to the estimation of winter wheat yield. Using the localized ALMANAC crop model, the climatic yield of winter wheat in the key growth period can be 
obtained, multiplied by $\mathrm{R}$ is the actual crop yield, and combined with the winter wheat planting area, the total output value of a county can be obtained (Table 1).

\section{Results and Analysis}

\subsection{Crop Acreage Verification}

The accuracy of the extraction of winter wheat acreage plays a crucial role in the prediction of subsequent yields. The extraction results of winter wheat area in southwestern Shanxi in 2015 and 2016 are shown in Figure 1. The distribution of winter wheat is basically the same as the actual situation. The planting area is mainly concentrated in Yuncheng and Linyi areas, and Changzhi and Jincheng are relatively few. Table 2 shows the relative error between the total planting area extracted by remote sensing and the statistics of the Ministry of Agriculture. The results show high accuracy and can be used for winter wheat yield estimation.

\subsection{Comparison with Measured Points}

In this paper, three types of high-, medium-, and low-yielding winter wheat planting areas were selected in Hongdong, and they were monitored and tracked in real time during the key growth period, and compared with the inversion monitoring results, the winter wheat high-yield area grew well and the middle-class area The growth is good, and the growth in the low-yield areas is generally the same as that of winter wheat (Figure 2).

\subsection{Crop Growth Monitoring Analysis}

The growth monitoring of the key growth period of winter wheat in 2015 and 2016 was selected separately, as shown in Figure 3. It can be seen from the figure that these two well-developed areas are mainly distributed in the northern part of Yuncheng Basin and the central part of Linfen Basin.

\subsection{Crop Yield Estimation Verification}

Using the winter wheat yield estimation model combined with remote sensing-crop model, the winter wheat harvesting period was selected in two key growth stages of winter wheat and the filling stage, and the yield of the image-wise

Table 1. Standards for the classification of growth monitoring.

\begin{tabular}{ccc}
\hline Grade & Division & Standard \\
\hline 1 & $0<\mathrm{R} \leq 0.2$ & More worse than normal \\
2 & $0.2<\mathrm{R} \leq 0.8$ & Slightly worse than normal \\
3 & $0.8<\mathrm{R} \leq 1.2$ & Equal to normal years \\
4 & $1.2<\mathrm{R} \leq 1.8$ & Slightly better than normal \\
5 & $\mathrm{R}>1.8$ & More better than normal \\
\hline
\end{tabular}


Table 2. Precision of winter wheat planting area.

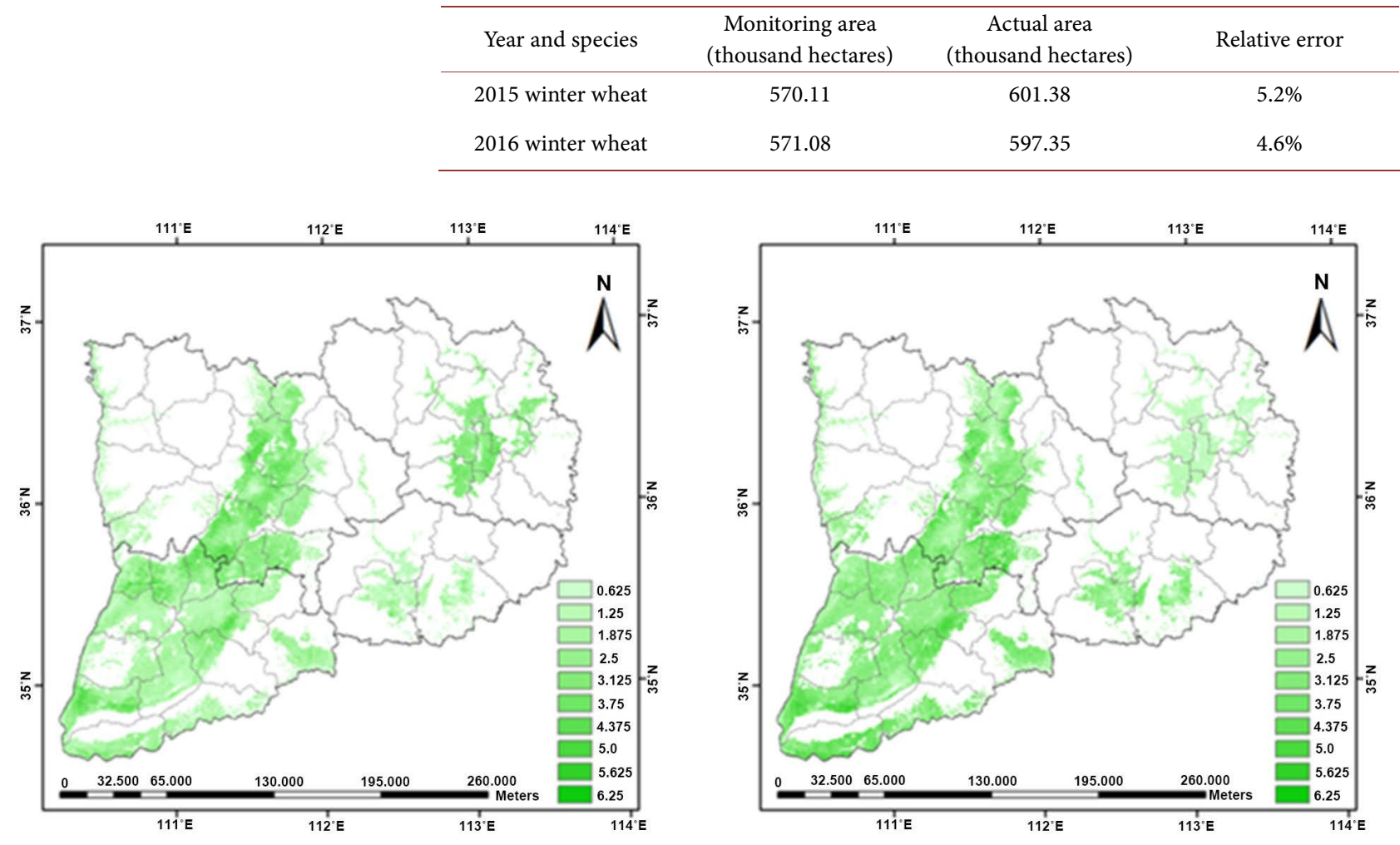

Figure 1. Area distribution of main wheat planting areas in Shanxi Province (On the left is 2015 and on the right is 2016).

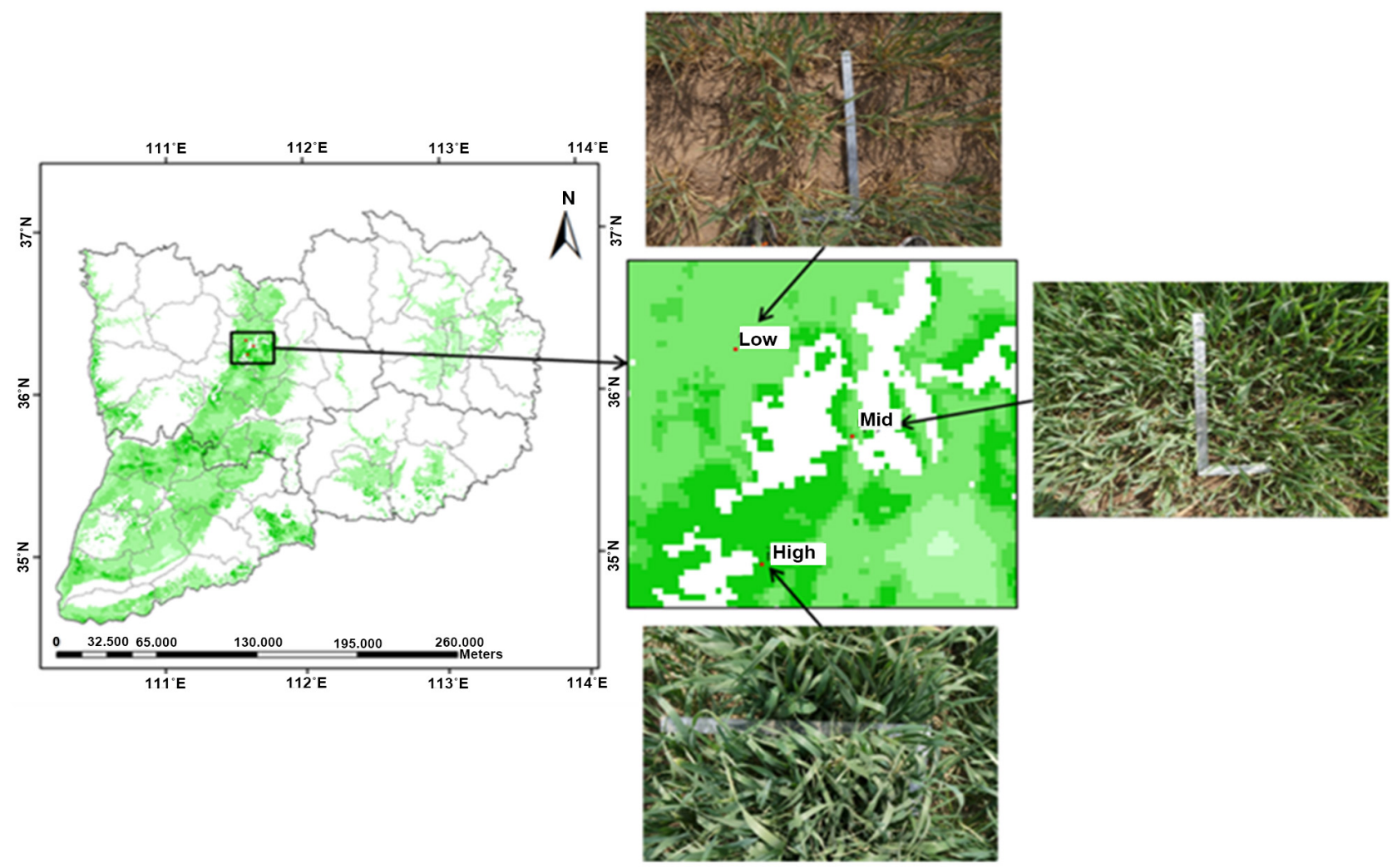

Figure 2. Comparison of the monitoring results of the winter wheat heading period in 2015 and the actual situation. 
winter wheat in 2015 and 2016 was estimated and compared with the statistics of the Ministry of Agriculture. Table 3 provides an accurate assessment of the estimated total winter wheat yield in southern Shanxi Province. In general, it is feasible to use the remote sensing-crop model method to predict the yield of winter wheat during the main growth period (late jointing stage and filling stage), and the yield prediction accuracy during the filling stage is higher than that in the late stage of jointing. During the filling period, the grain has been formed. If there is no dry hot wind, heavy rainfall and other disasters, the external environment (light, temperature, precipitation) has little effect on crop yield, and the prediction result is more stable.

\section{Conclusion and Discussions}

Before the study of this paper, some research results have been accumulated on the winter wheat planting area extraction and ALMANAC crop model localization. Therefore, the focus of this paper is to combine remote sensing and crop models to achieve real-time and dynamic monitoring from point to point. The growth situation of winter wheat in Shanxi Province and the estimation of production yielded the following conclusions:

1) Compared with MODIS data, MERSI data has higher spatial resolution. This paper combines MERSI data with ALMANAC crop model to achieve growth monitoring and yield estimation for winter wheat growing areas in southern Shanxi Province, and improve spatial resolution.

2) The winter wheat NDVI-LAI model based on NDVI time series can better combine MERSI data with crop model to realize the acquisition of LAI from point to surface, which lays a foundation for later growth monitoring and yield estimation research.
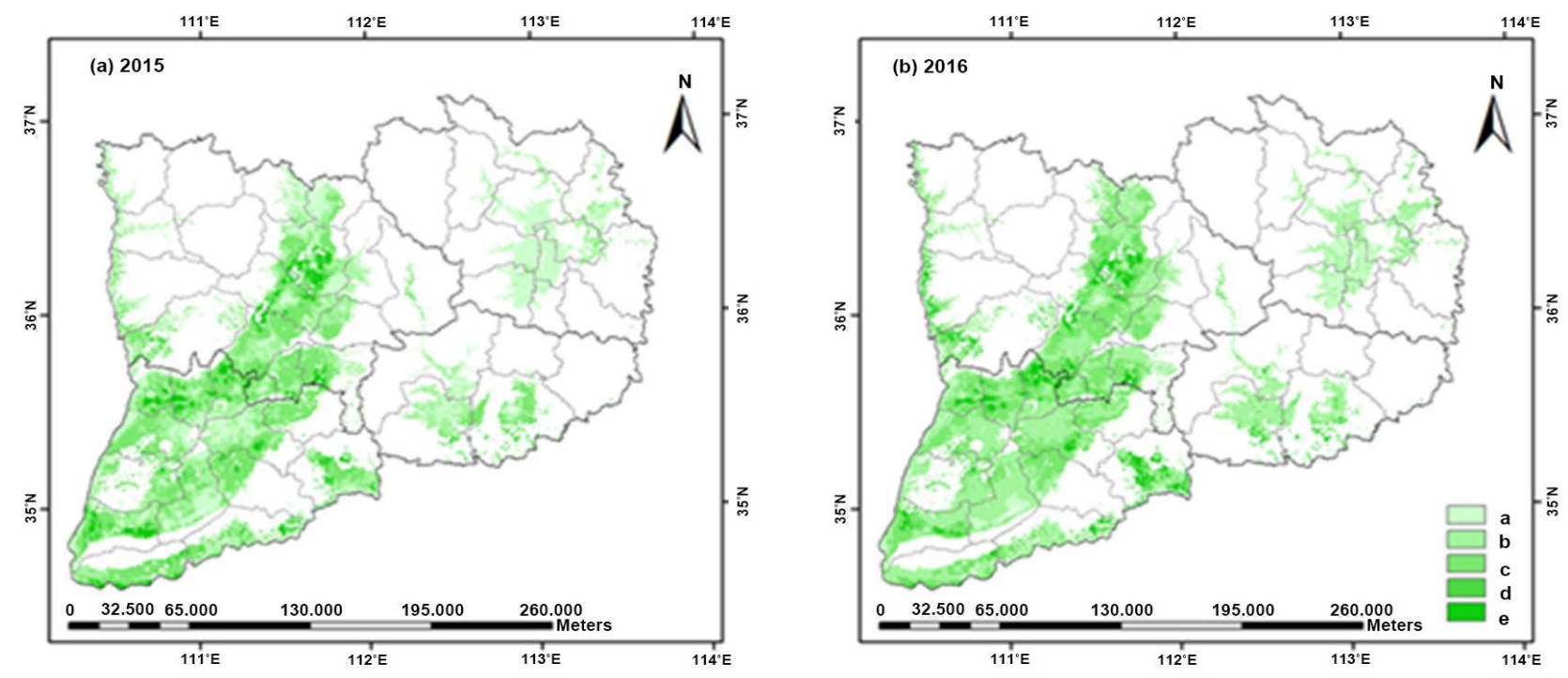

Figure 3. Real-time monitoring of winter wheat heading stage in southern Shanxi Province (a, b, c, d and e represents worse than normal, slightly worse than normal, equal to normal years, slightly better than normal and more better than normal, respectively). 
Table 3. Prediction accuracy of total winter wheat yield in southern Shanxi Province.

\begin{tabular}{ccccc}
\hline $\begin{array}{c}\text { Year and } \\
\text { species }\end{array}$ & Growth period & $\begin{array}{c}\text { Actual statistical } \\
\text { output }\left(10^{4} \text { tons }\right)\end{array}$ & $\begin{array}{c}\text { Model estimated } \\
\text { output }\left(10^{4} \text { tons }\right)\end{array}$ & Relative error \\
\hline $\begin{array}{c}2015 \\
\text { winter wheat }\end{array}$ & Late jointing & 291 & 267.98 & $7.91 \%$ \\
& Filling period & & 274.59 & $5.64 \%$ \\
2016 & Late jointing & 278 & 297.738 & $7.10 \%$ \\
winter wheat & Filling period & & 291.64 & $4.91 \%$ \\
\hline
\end{tabular}

3) LAI is an important agronomic parameter for growth monitoring and yield estimation. Based on LAI, the winter wheat growth monitoring and yield estimation model is established, and the model is applied to the growth of winter wheat in the key growth period of southern Shanxi in 2015 and 2016 respectively. It was used for monitoring and production estimates and found that the yield prediction result in the filling stage is closest to the actual statistical output value, and the relative error of total yield is about $5 \%$, indicating that the combination of crop model and satellite remote sensing is predictive of crop yield, and the formation of wheat is good or bad. Determining the fundamental factors of grain production, and selecting the grain filling period to predict the output is more accurate.

In this paper, when introducing the ALMANAC crop model, this paper will use $\mathrm{Wu}$ Yongli and other based on the adjustment parameters of a single site in Hongdong County, which should be in the entire southwestern research area. Although the characteristics of winter wheat growth and development in Hongdong area are representative in the southwestern part of the mountain, it does not fully represent the winter wheat development characteristics of the whole study area. In future research, we will adjust the model parameters adapted to different cities or counties, thereby improving the accuracy of the potential monitoring and yield estimation on the pixel scale. In addition, from the results of this study, the error caused by the area estimation will increase further in the production forecast, so the area estimation accuracy will be further improved in the future.

We suggest in this article that the morphological and agricultural situation upon the total winter yield in southern Shanxi Province, China has been given in a fairly well developed manner. However, it is not clear why the exceptional novel features are derived from the ALMANAC crop model. The important role of the local government, agricultural education and public intervention might be studied by philosophers, economists and social scientists. Advances in agricultural technology, the cultivation of new varieties, and the conclusions of this paper apply to other provinces in China, which can be a focus of our future research. In addition, the impact of crop varieties (Liu et al., 2019), soil N, K, P fertility (Zhang et al., 2018; Zhao et al., 2016), meteorological factors such as temperature and precipitation (Dong et al., 2018; Liu et al., 2013) on agricultural 
production can also be used as a research focus in the future.

\section{Conflicts of Interest}

The authors declare no conflicts of interest regarding the publication of this paper.

\section{References}

Clevers, J. G. P. W., Büker, C., Leeuwen, H. J. C. V., \& Bouman, B.A.M. (1994). A Framework for Monitoring Crop Growth by Combining Directional and Spectral Remote Sensing Information. Remote Sensing of Environment, 50, 161-170.

https://doi.org/10.1016/0034-4257(94)90042-6

Dong, B., Yang, H., Qiao, Y., Zhang, M., Wang, Y., Jin, L., \& Liu, M. (2018). Different Growing Strategies of Two Winter Wheat Cultivars under Rainfed Conditions during Dry Years in North China Plain. International Journal of Agricultural and Biological Engineering, 11, 150-159. https://doi.org/10.25165/j.ijabe.20181105.4344

Gao, Z., \& Liu, J. (2000). Research on Driving Factors and Models of NDVI Change in China Based on Remote Sensing and GIS. Climate and Environment Research, 5, 155-164.

Guerif, M., \& Duke, C. (1998). Calibration of the SUCROS Emergence and Early Growth Module for Sugar Beet Using Optical Remote Sensing Data Assimilation. European Journal of Agronomy, 9, 127-136. https://doi.org/10.1016/S1161-0301(98)00031-8

Jiang, Z. (2012). Research on Remote Sensing Data Assimilation Technology for Regional Winter Wheat Yield Estimation. Doctoral Thesis, Chinese Academy of Agricultural Sciences, Beijing.

Kiniry, J. R., Asnderson, M. A., Williams, J. R. et al. (1996). Simulating Alamo Switch Grass with the ALMANAC Model. Agronomy Journal, 88, 602-606.

https://doi.org/10.2134/agronj1996.00021962008800040018x

Kiniry, J. R., Williams, J. R., \& Gassman, P. W. (1992). A General, Process-Oriented Model for Two Competing Plant Species. Transactions of the ASAE, 35, 801-810. https://doi.org/10.13031/2013.28665

Li, D. (1997). On the Definition, Theory and Key Technologies of RS, GPS and GIS Integration. Journal of Remote Sensing, 1, 64-68.

Liu, L., Yao, S., Zhang, H., Muhammed, A., Xu, J., Li, R., Zhang, D., Zhang, S., \& Yang, X. (2019). Soil Nitrate Nitrogen Buffer Capacity and Environmentally Safe Nitrogen Rate for Winter Wheat-Summer Maize Cropping in Northern China. Agricultural Water Management, 213, 445-453. https://doi.org/10.1016/j.agwat.2018.11.001

Liu, Q., Mei, X. R., Yan, C. R., Ju, H., \& Yang, J. Y. (2013). Dynamic Variation of Water Deficit of Winter Wheat and Its Possible Climatic Factors in Northern China. Acta Ecologica Sinica, 33, 6643-6651. https://doi.org/10.5846/stxb201212151804

Mohamed, M. A. A., Babiker, I. S., Chen, Z. M. et al. (2004). The Role of Climate Variability in the Inter-Annual Variation of Terrestrial Net Primary Production (NPP). Science of the Total Environment, 332, 123-137. https://doi.org/10.1016/j.scitotenv.2004.03.009

Moulin, S., Fischer, A., \& Dedieu, G. (1995). Interannual Variations of Radiometric Time Profile and Crop Yield: Coupling of Functional Model and Short Wavelength Remote Sensing Observations. In Proceedings of 1995 International Geoscience and Remote Sensing Symposium (Vol. 2, pp. 1468-1470). Firenze, Italy. 
Ren, J., Chen, Z., Zhou, Q. et al. (2010). Remote Sensing Estimation of Regional Winter Wheat Yield Based on Leaf Area Index Inversion. Chinese Journal of Applied Ecology, 221, 2883-2888.

Wang, L., Chen, X., Li, Q. et al. (2017). Effects of Different Time Series Vegetation Index Reconstruction Methods on the Phenological Extraction of Winter Wheat in Jiangsu Province. Science Technology and Engineering, 17, 192-199.

Wang, L., Zhang, Y., Li, Q. et al. (2018). Current Status and Prospects of Sensitivity Analysis of Crop Model Parameters. Meteorological Science and Technology, 2, 382-389.

Wu, B., Zhang, F., Liu, C. et al. (2008). Integrated Remote Sensing Monitoring Method for Crop Growth. Journal of Remote Sensing, 6.

Wu, Y., Wang, Y., Zhang, J. et al. (2009). Remote Sensing of Winter Wheat Planting Area Using Linear Mixed Model. Transactions of the Chinese Society of Agricultural Engineering, 25, 136-140.

Wu, Y., Yan, Q., \& Tian, G. (2011). Atmospheric Correction of FY-3A/MERSI Visible to Near-Infrared Based on 6S Model. Chinese Journal of Applied Ecology, 22, 1537-1542.

Wu, Y., Zhao, Y., Xiang, D. et al. (2018). Simulation of Winter Wheat in Shanxi Province Based on ALMANAC. Chinese Agricultural Science Bulletin, 34, 134-138.

Zhang, J. (2012). Estimation of Winter Wheat Area in Jining City, Shandong Province Based on MODIS Remote Sensing Data. Nanjing: Nanjing University.

Zhang, J. J., He, P., Xu, X. P., Ding, W. C., Ullah, S., Wang, Y. L., Jia, L. L., Cui, R. Z., Wang, H. T., \& Zhou, W. (2018). Nutrient Expert Improves Nitrogen Efficiency and Environmental Benefits for Winter Wheat in China. Agronomy Journal, 110, 696-706. https://doi.org/10.2134/agronj2017.05.0291

Zhao, H. B., Wang, Z. H., Xue, C., Huang, D. L., \& Malhi, S. S. (2016). Increased Dryland Winter Wheat Yields by Nitrogen Fertilizer Topdressing and Its Relationship to Soil Moisture, Available N, P and $\mathrm{K}$ in Northwestern China. Communications in Soil Science and Plant Analysis, 47, 543-553. https://doi.org/10.1080/00103624.2016.1141917

Zhao, Y., Zhao, H., \& Xu, F. et al. (2004). Interpretation of Winter Wheat Yield Type Distribution Using Satellite Remote Sensing Information. Meteorological Science and Technology, 32, 57-59.

Zhou, H., Wang, N., Huang, Y. et al. (2016). Comparative Analysis of Remote Sensing Time Series Reconstruction Models at Different Time Intervals. Journal of Earth Sciences, 18, 1410-1417. 